\title{
Differential secretome of pancreatic cancer cells in serum-containing conditioned medium reveals CCT8 as a new biomarker of pancreatic cancer invasion and metastasis
}

\author{
Peng Liu' ${ }^{1}$ Lingming Kong ${ }^{1}$, Haoyi Jin ${ }^{1}$, Yunhao Wu ${ }^{1}$, Xiaodong Tan ${ }^{1 *+}$ and Bing Song ${ }^{1,2^{*+}}$
}

\begin{abstract}
Background: Pancreatic cancer is a malignancy with a very poor prognosis. The emergence of liquid biopsy is expected to achieve accurate early diagnosis through detection of tumor-derived secreted proteins in the blood. Early diagnosis and treatment of pancreatic cancer could help to improve prognosis.

Methods: The pretreatment approach of samples can have a major effect on downstream analysis. In this study, we used a pair of homologous pancreatic cancer cell supernatants with different capacities for invasion and metastasis to examine secreted proteins in the conditioned media without the removal of fetal bovine serum, namely through size exclusion chromatography combined with high-abundance protein affinity chromatography to enrich lowconcentration protein, followed by mass spectrometry using triple dimethyl labeling. Identification of proteins was performed using an online public database and western blot.

Results: Mass spectrometry data revealed 77 proteins with quantitative properties, of which 12 proteins had over a 1.5-fold difference (in the supernatant of the highly invasive pancreatic cancer cell line PC-1.0, the expression of 8 proteins were increased and the expression of 4 proteins were decreased). Bioinformatics analysis results showed that CCT8, CTSL, SAA1, IGF2 are secreted via the exosome pathway. According to the literature, with the exception of CCT8, the other three proteins can be detected in blood samples of pancreatic cancer patients, and they can be used as prognostic markers. Western blot results were used to validate consistency with MS results.
\end{abstract}

Conclusion: This study found that CCT8 can be used as a liquid biopsy marker to assess the prognosis of pancreatic cancer patients.

Keywords: CCT8, Pancreatic cancer, Serum-containing conditioned medium, Biomarker

\section{Background}

Pancreatic cancer ranks fourth in cancer mortality in the United States. In 2019, 56,770 new cases of pancreatic cancer are expected in the United States. Due to its anatomical location deep in the abdominal cavity, patients

\footnotetext{
*Correspondence: tanxdcmu@163.com; songb@cardiff.ac.uk

${ }^{\dagger}$ Xiaodong Tan and Bing Song contributed equally to this work and should be considered co-corresponding authors

1 1st Department of General Surgery, Shengjing Hospital, China Medical University, Shenyang 110004, China

${ }^{2}$ Cardiff Institute of Tissue Engineering and Repair, School of Dentistry, Cardiff University, Cardiff CF14 4XY, UK
}

do not present unique symptoms, and most tumors are at an advanced stage at the time of diagnosis and are often accompanied by vascular invasion or distant metastasis. Surgical treatment is an option in only $15-20 \%$ of pancreatic cancer patients, and the overall 5-year survival rate is less than $5 \%[1,2]$. Early diagnosis and appropriate treatment can significantly improve the prognosis of pancreatic cancer. With the advancement of experimental techniques, the number of molecular and chemical detection methods for pancreatic cancer has increased, and these methods have played an important role in the early diagnosis of pancreatic cancer [3]. 
Tumor microenvironment can regulate cancer development and metastasis. Hanahan et al. have reported that heterogeneous signaling pathways between the parenchyma and mesenchyme of a tumor play an important role in tumor invasion and metastasis [4]. Studies have confirmed that secreted proteins play an important role in the tumor microenvironment [5]. In previous studies, we used two cell lines with distinct capacities for invasion and metastasis: a non-dissociated, weakly metastatic pancreatic cancer cell line (PC-1) and a dissociated, highly metastatic pancreatic cancer cell line (PC-1.0). PC-1.0 cell supernatant was purified, prepared into a conditioned medium, and used for culture of the weakly metastatic PC-1 cells. It was found that the growth of PC-1 cells changed to the growth pattern of PC-1.0. Therefore, we concluded that PC-1.0 supernatant contains key factors that promote changes in the biological behavior of the cells [6].

In this study, the secretome was extracted from the serum-containing supernatant, and low-abundance proteins were enriched by online chromatographic separation. In addition, the pancreatic cancer secretome was analyzed in combination with triple dimethyl labeling. 77 proteins were identified, of which 12 with a fold change of over 1.5 were considered differentially expressed proteins. DAVID and STRING software were used to analyze the function of differential proteins and their possible interacting proteins. Survival analysis of these differential genes was performed using a public pancreatic cancer database. Finally, we selected CCT8 and CTSL for protein validation in two pairs of cell lines with different capacities for invasion and metastasis.

\section{Materials and methods}

\section{Instruments, reagents, and materials}

Acetonitrile (ACN) and methanol were purchased from Merck (Germany). Glacial acetic acid was purchased from Damao Chemical Reagent Factory (Tianjin, China). Bovine serum albumin (BSA), formaldehyde and its isotopic reagents $\left(\mathrm{CH}_{2} \mathrm{O},{ }^{13} \mathrm{CH}_{2} \mathrm{O},{ }^{13} \mathrm{CD}_{2} \mathrm{O}, \mathrm{CD}_{2} \mathrm{O}\right)$, sodium cyanoborohydride $\left(\mathrm{NaBH}_{3} \mathrm{CN}\right)$, deuterated sodium cyanoborohydride $\left(\mathrm{NaBD}_{3} \mathrm{CN}\right)$, heavy water $\left(\mathrm{D}_{2} \mathrm{O}\right)$, $\alpha$-cyano4-hydroxycinnamic acid (CHCA), and trifluoro acetic acid (TFA) were purchased from Sigma-Aldrich (USA). All water for experiments was purified using the Milli-Q system (Millipore, USA). The centrifuge was purchased from Sigma (USA). The Thermo SEC120 HPLC column (size $5 \mu \mathrm{m}, 120 \AA$ ) and Agilent Human 14 Multiple Affinity Removal Column (H14, size: $10 \mathrm{~mm} \times 100 \mathrm{~mm}$ ) were used. The Ultimate 3000 chromatography instrument was used with the Thermo LTQ-Orbitrap mass spectrometer for detection.

\section{Cell culture}

PC-1.0 and PC-1 cells were given friendly by Kumamoto University Medical School (Kumamoto, Japan) and the use of these cell lines was approved by China Medical University Affiliated Shengjing Hospital Medical Research. Aspc-1, Capan-2 cells, and HPDE6-C7 were purchased from the Institute of Biochemistry and Cell Biology, Chinese Academy of Sciences (Shanghai, P.R. China). PC-1 cells grew as islet-like clones of cells, whereas PC-1.0 cells grew as single cells. These cells were cultured in RPMI-1640 (Gibco-BRL, Grand Island, NY) supplemented with $10 \%$ fetal bovine serum $+1 \%$ streptomycin. Cells were incubated in a $37{ }^{\circ} \mathrm{C}$ incubator with $5 \% \mathrm{CO}_{2}$. Cells were passaged when they reached a confluence of about $80 \%$ [7].

\section{Experimental methods \\ Extraction of total protein from samples}

PC-1.0 and PC-1 cells were cultured in complete growth medium until $80-90 \%$ confluent. The supernatant was collected and protease inhibitors were added. The supernatant was centrifuged at $500 \mathrm{~g}$ and $3000 \mathrm{~g}$ for $15 \mathrm{~min}$ to remove debris. The mixture was spun at $12000 \mathrm{r} / \mathrm{min}$ through a $0.22 \mu \mathrm{m}$ fiber filter and concentrated using a $3 \mathrm{kDa}$ concentrating tube by centrifuging at $3500 \mathrm{~g}$ for $120 \mathrm{~min}$. Protein samples included three independent biological replicates. Protein concentration was measured using the BCA method. The experimental process is shown in Fig. 1.

\section{SEC and H14 sample isolation}

High-abundance proteins were removed using a size exclusion chromatography (SEC) column combined with an Agilent multiple affinity removal spin cartridge (H14) to enrich low-abundance proteins. A $200 \mu \mathrm{L}$ sample was injected and washed with buffer $\mathrm{A}$ at a flow rate of $0.5 \mathrm{~mL} / \mathrm{min}$ for $10 \mathrm{~min}$. After the flow through fractions were collected, the remaining fractions were eluted with buffer B at $1 \mathrm{~mL} / \mathrm{min}$ for $7 \mathrm{~min}$. The collected fractions were stored at $-20{ }^{\circ} \mathrm{C}$ until use. The flow through fractions collected from the injection were concentrated in a rotary concentrator with a $5 \mathrm{kDa}$ molecular weight cutoff membrane. The sample was centrifuged at $5000 \mathrm{r} / \mathrm{min}$ at $10^{\circ} \mathrm{C}$.

\section{Triple dimethyl labeling}

Protein concentration was measured using the $\mathrm{BCA}$ method, and samples were prepared using FASP. PC-1.0 cells were labeled with $50 \mathrm{mM}$ pH 8.0 phosphate buffer with $20 \mu \mathrm{L}$ of $4 \%$ formaldehyde and $20 \mu \mathrm{L}$ of $0.6 \mathrm{M}$ sodium cyanoborohydride (light label) added. PC-1 cells were labeled with $20 \mu \mathrm{L} 4 \%$ deuterated formaldehyde and $20 \mu \mathrm{L}$ of $0.6 \mathrm{M}$ sodium cyanoborohydride (medium 


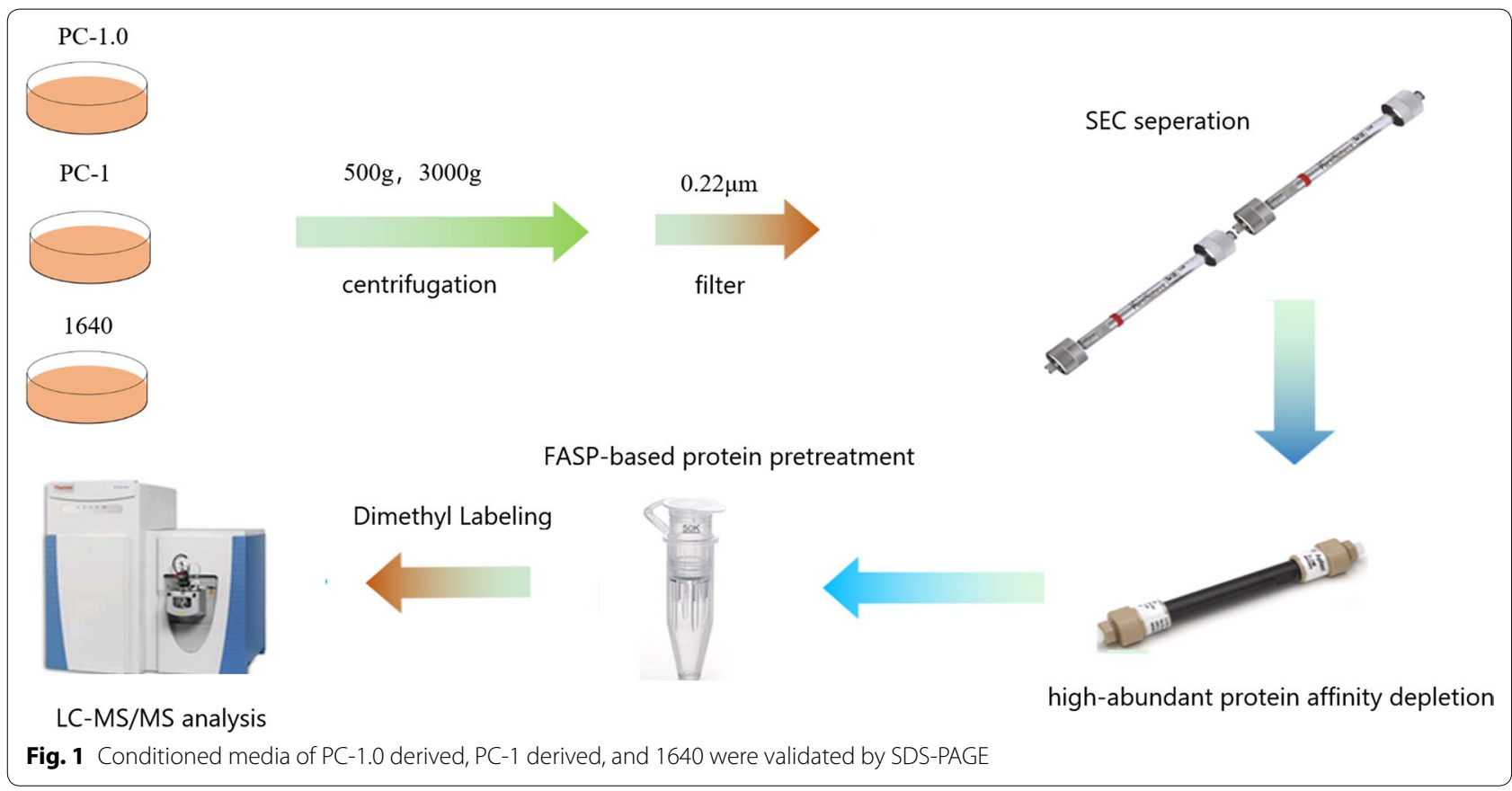

label). 1640 medium was labeled with $20 \mu \mathrm{L}$ of $4 \%$ ${ }^{13} \mathrm{CD}_{2} \mathrm{O}$ and $20 \mu \mathrm{L}$ of $0.6 \mathrm{M}$ deuterated sodium cyanoborohydride (heavy label). The reaction was conducted at room temperature for $1 \mathrm{~h}$. Labeled solutions were combined, lyophilized and stored at $-20{ }^{\circ} \mathrm{C}$.

\section{Capillary liquid chromatography-mass spectrometry analysis and database search}

The Ultimate 3000 chromatography instrument was used with the Thermo LTQ-Orbitrap mass spectrometer. The flow direction was divided into an A phase $\left(98 \% \mathrm{H}_{2} \mathrm{O}\right.$ and $2 \% \mathrm{ACN}$ with $0.1 \%$ formic acid) and a $\mathrm{B}$ phase $(2 \%$ $\mathrm{H}_{2} \mathrm{O}$ and $98 \%$ ACN with $0.1 \%$ formic acid). We used a custom C18 capillary trap column $(150 \mu \mathrm{m}$ inner diameter $\times 4 \mathrm{~cm})$ and a separation column $(75 \mu \mathrm{m}$ inner diameter $\times 15 \mathrm{~cm}$ ) with a flow rate of $120 \mu \mathrm{L} / \mathrm{min}$. Mobile phase setting: $0-6 \% \mathrm{~B}$ phase for $10 \mathrm{~min}$, then $6-35 \% \mathrm{~B}$ phase for $100 \mathrm{~min}, 35-80 \% \mathrm{~B}$ phase for $10 \mathrm{~min}$, and finally $80 \%$ $\mathrm{B}$ phase for $10 \mathrm{~min}$. The voltage was set to $2.7 \mathrm{kV}$ and the ion transfer capillary temperature was $275^{\circ} \mathrm{C}$. Mass spectrometry was sequenced in a data-dependent manner. The CID ion dissociation method was used, a dynamic 20-second exclusion window was set, and the mass spectrometry ion mass-to-charge ratio scan range was from 300 to 1800 . Samples were measured in triplicate. The experimentally obtained mass spectra were searched using Maxquant software to analyze the data files (.RAW) produced by the mass spectrometry. Settings for relevant parameters: mass error of the parent ion was $7 \mathrm{ppm}$, and mass error of the daughter ion was $20 \mathrm{mmu}$. The fixation modification was an alkylation modification to cysteine, and the variable modification is an oxidation modification of methionine. The maximum number of missed sites allowed was 2, and the FDR for peptide and protein identification was less than $1 \%$.

\section{Bioinformatics analysis}

In this study, quantitative data was obtained for all three experiments, the $p$-value was $<0.05$, indicating that the data was reliable. We considered fold differences greater than 1.5 to be differential expression. To elucidate the function of secreted proteins, we performed bioinformatics analysis using DAVID (http://david.abcc.ncifcrf.gov/) [8] and STRING (https://string-db.org/) [9] software. In addition, we also searched the Exocarta database [10] to determine if these proteins were exosomes. Finally, we performed a matching search on pancreatic cancer genomics studies publicly available so far, and observed the association of genes corresponding to these proteins with pancreatic cancer [11].

\section{Sodium dodecyl sulfate polyacrylamide gel electrophoresis (SDS-PAGE)}

To an appropriate amount of protein sample solution, an equal volume of reducing loading buffer (2\% SDS, $20 \%$ glycerol, $20 \mathrm{mmol} / \mathrm{L}$ Tris-HCl, pH 6.8, $6 \mathrm{mmol} / \mathrm{L}$ ß-mercaptoethanol, a trace amount of bromophenol blue) was added. After boiling for $5 \mathrm{~min}$, separation was performed using a vertical electrophoresis apparatus. After the 
electrophoresis was finished, the film was stained with silver nitrate and scanned.

\section{Western blot}

Western blotting was performed as described previously [7]. Primary antibodies against CCT8 polyclonal antibody (Abcam, USA) and CTSL polyclonal antibody (Proteintech, USA) were used. Supernatant was collected when the cells reached $80-90 \%$ confluence, and protease inhibitors were added. The supernatant was centrifuged at $500 \mathrm{~g}$ and $3000 \mathrm{~g}$ for $15 \mathrm{~min}$, the supernatant was collected, proteins were concentrated using a $3 \mathrm{kDa}$ concentrating tube, and protein concentration was measured using the BCA method. Samples of equivalent total protein $(20 \mu \mathrm{g})$ were loaded.

\section{Cell transfection}

shRNA plasmid specific for CCT8, scrambled shRNA, and lentiviral vector were purchased from GeneChem (GeneChem, Shanghai, China). Stable transfection with lentiviral vector was performed in accordance with manufacturer's protocols. Scrambled shRNA was used as control.

\section{Cell invasion and migration assays}

Wound healing migration assay and Transwell invasion assay were performed as previously described [7].

\section{Results}

\section{Immunoblotting detection of pancreatic cancer supernatant}

In order to initially detect overall protein content in the supernatant of pancreatic cancer cells, we collected total protein from the supernatant and performed SDS-PAGE electrophoresis and silver staining (Fig. 2). The results showed that the approximate distribution of proteins in the supernatant of pancreatic cancer cells: high molecular weight proteins (greater than $44 \mathrm{kDa}$ ) were abundant, and there were fewer low molecular weight proteins (less than $20 \mathrm{kDa}$ ).

\section{Proteins with quantitative differential expression}

The experimental samples were separated using SEC and $\mathrm{H} 14$ columns to enrich the concentration of low abundance proteins. A total of 77 proteins were identified using Maxquant software through the combined calculations of three experiments (Additional file 1: Table S1 and Additional file 2: Table S2). Functional annotation analysis of these proteins using DAVID revealed close association with cell secretion, cell proliferation, and migration and metastasis (Table 1). In the experiment, we performed triple dimethyl labeling, namely, supernatants of highly invasive PC-1.0 cells with a light

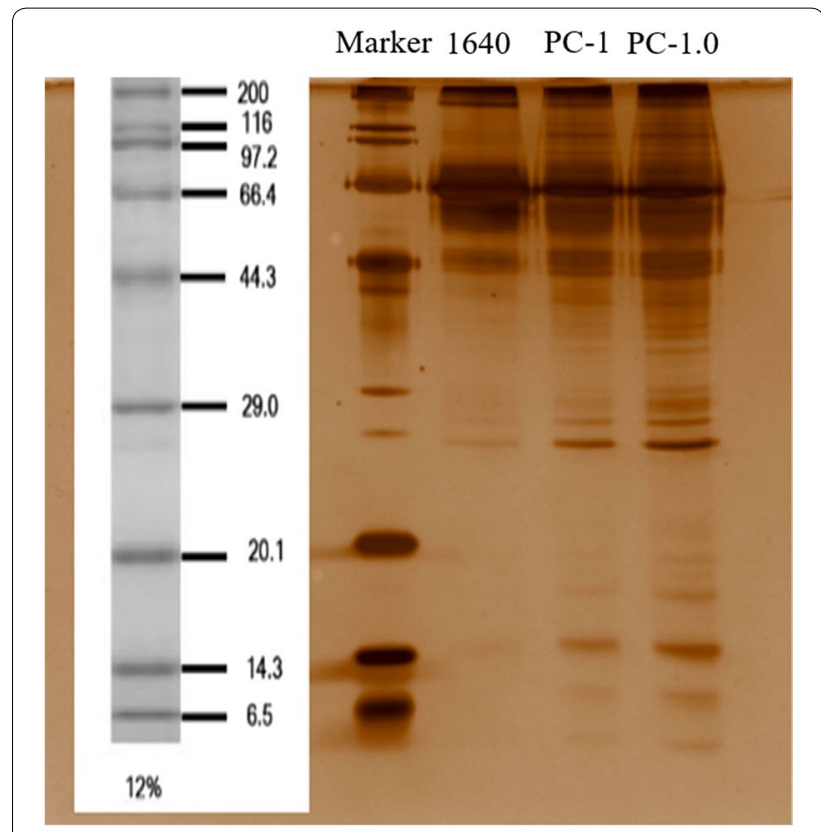

Fig. 2 PPI network analysis of differentially expressed secretome proteins from $P C-1.0$ and $P C-1$ cells

label, supernatants of weakly invasive PC-1 cells with a medium label, and 1640 medium with a heavy standard. By labeling the 1640 medium, we removed interference from serum proteins in the medium, which enhanced the reliability of the identification results. Based on the results of the search, we considered proteins with an over 1.5 -fold change in the ratio of the three labels as significant differentially expressed proteins. The results showed that there were 12 differentially expressed proteins, of which 8 were up-regulated and 4 were down-regulated (Table 2).

\section{Gene ontology, KEGG, and PPI analysis}

GO analysis was performed using online DAVID software. The primary enrichment in cellular component (CC) was cytoplasmic vesicle and extracellular exosome, and the primary enrichment in biological process (BP) was the regulation of cell adhesion. The primary enrichment in molecular function (MF) was protein complex binding. Using functional annotation clustering analysis in the software, we found that the primary cluster was exosome signaling, which included four proteins (CTSL, CCT8, IGF2 and SAA1). These results also confirmed that we identified secreted proteins (Additional file 3: Table S3). KEGG analysis showed that proteins were concentrated on the proteoglycans in cancer $(p=0.0025)$ signaling pathway. To further analyze the role of the identified proteins, we used the STRING database to search for DEPs. The results 
Table 1 DAVID revealed close association with cell secretion, cell proliferation, and migration and metastasis

\begin{tabular}{|c|c|c|c|}
\hline Category & Term & P-value & Fold enrichment \\
\hline Annotation Cluster 1 & Enrichment Score: 15.7 & & \\
\hline UP_KEYWORDS & Secreted & $5.81 E-26$ & 12.93408 \\
\hline Annotation Cluster 2 & Enrichment Score: 3.3 & & \\
\hline GOTERM_MF_DIRECT & GO:0031994 insulin-like growth factor I binding & $1.29 \mathrm{E}-04$ & 163.6875 \\
\hline Annotation Cluster 3 & Enrichment Score: 3.0 & & \\
\hline KEGG_PATHWAY & bta04610:Complement and coagulation cascades & $6.54 \mathrm{E}-05$ & 21.25563 \\
\hline Annotation Cluster 4 & Enrichment Score: 2.6 & & \\
\hline UP_KEYWORDS & Extracellular matrix & $1.02 \mathrm{E}-05$ & 20.19811 \\
\hline Annotation Cluster 5 & Enrichment Score: 2.5 & & \\
\hline GOTERM_CC_DIRECT & GO:0005604 basement membrane & 0.009647 & 19.7711 \\
\hline Annotation Cluster 6 & Enrichment Score: 1.9 & & \\
\hline KEGG_PATHWAY & bta04510:Focal adhesion & 0.003284 & 7.562099 \\
\hline Annotation Cluster 7 & Enrichment Score: 1.8 & & \\
\hline UP_SEQ_FEATURE & Domain:Peptidase S1 & 0.002328 & 14.57442 \\
\hline
\end{tabular}

Table 2 List of 12 proteins differentially expression by PC-1.0 and PC-1 pancreatic cancer cells with fold change $>1.5$

\begin{tabular}{llcc}
\hline Protein & $\begin{array}{l}\text { Ratio L/M } \\
\text { (PC-1.0/PC-1) }\end{array}$ & $\begin{array}{l}\text { Ratio L/H } \\
\text { (PC-1.0/1640) }\end{array}$ & $\begin{array}{l}\text { Ratio M/H } \\
\text { (PC-1/1640) }\end{array}$ \\
\hline SAA1 & 6.96 & 1.56 & 0.22 \\
Roquin-1 & 3.29 & 1.52 & 0.55 \\
CCT8 & 2.74 & 1.91 & 0.63 \\
ZNF518B & 2.14 & 4.39 & 1.85 \\
CTSL & 1.77 & 17.12 & 10.46 \\
EXOSC8 & 1.60 & 3.16 & 2.57 \\
IGF2 & 1.61 & 0.64 & 0.43 \\
C40rf470 & 1.62 & 0.47 & 0.32 \\
Npc2 & 0.57 & 8.55 & 13.36 \\
HSP90AA1 & 0.45 & 0.66 & 1.54 \\
PPIA & 0.10 & 1.80 & 18.16 \\
ENO1 & 0.05 & 1.89 & 31.58 \\
\hline
\end{tabular}

showed that there is currently no literature support for interaction between the eight proteins, which may be due to the small number of proteins we identified. Therefore, we used the increased protein interaction function built into the software. It was found that as the software gradually increased protein interaction, SAA1 and CCT 8 could interact through GNB1, CCT8 and EXOSC8 were linked by EXOSC3, and CTSL and IGF2 were linked by IGFGB3. Therefore, through data mining, we believe that $\mathrm{CCT} 8$ may be a key protein in this PPI network (Fig. 3, Additional file 4: Table S4).
Possible clinical significance of differential proteins

We used the Human Protein Atlas database to search CCT8, CTSL, IGF2, and SAA1 for clinical correlation analysis. Using clinical data on pancreatic cancer provided by Pathology Atlas, we analyzed the FRKM values of the four genes and found that CCT8 and IGF2 were associated with clinical prognosis $(p=0.018$, $p=0.0001$ ) (Fig. 4, Additional file 5: Table S5). There have been no literature reports of the function of CCT8 in pancreatic cancer. We searched the Exocarta database and our results from exosome studies, as well as these four proteins [12], which revealed from a different perspective that the four secreted proteins were secreted outside the cell in the form of exosomes. According to currently available public data, these four secreted proteins are present in human plasma samples and can be used as liquid biopsy markers in the future.

Western blot validation of the expression of differentially expressed proteins in cell lines with different capacities for invasion and metastasis

We used two pairs of cell lines (PC-1.0 vs. PC-1 and Aspc-1 vs. Capan-2) to measure intracellular CTSL and CCT 8 protein expression. The results showed that CTSL and CCT8 were highly expressed in the highly invasive PC-1.0 and Aspc-1 cell lines (Fig. 5). This result is consistent with mass spectroscopy.

\section{Knockdown of CCT8 suppressed the migration and invasion capabilities}

To investigate the potential role of CCT8 in the metastasis of pancreatic cancer, we knocked down 


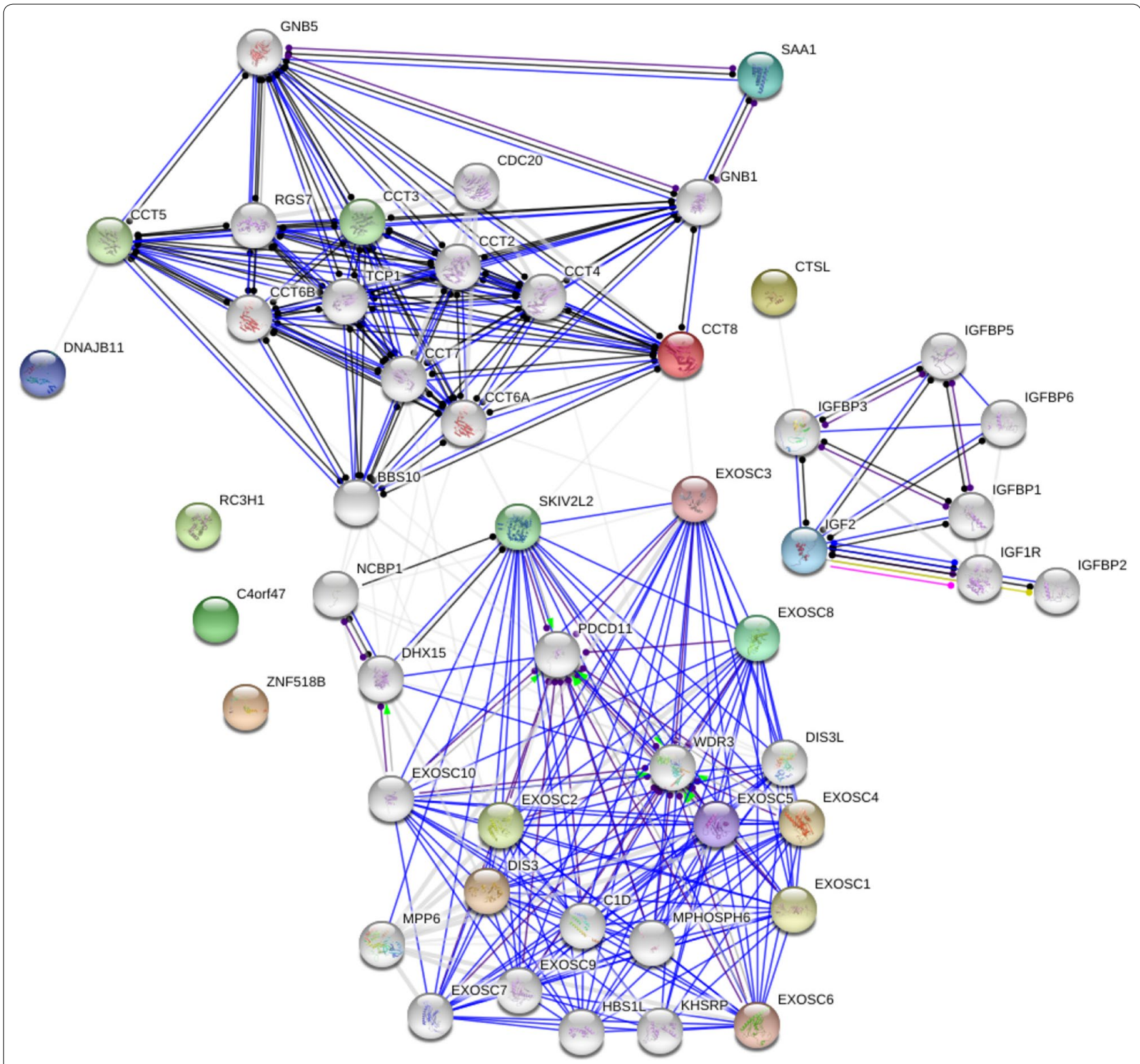

Fig. 3 CCT8 is a key protein in this network

the expression of CCT8 in PC-1.0 and AsPC-1 cell lines. In vitro migration and invasion assay showed that knockdown of CCT8 suppressed the migration and invasion capabilities of PC-1.0 and AsPC- 1 cells (Figs. 6, 7). These results suggested that knockdown of CCT8 could suppress the metastatic phenotype of these two pancreatic cancer cell lines.

\section{Discussion}

When tumor cells secrete proteins into the extracellular environment, some of these proteins can alter the tumor microenvironment to promote tumor growth [13].
Previous studies have also confirmed some tumor biomarkers through secretome analysis. Most proteomic studies were performed in serum-free medium. These studies have used serum starvation to reduce interference from a large number of serum proteins and have found many biomarkers, but many significant limitations still exist $[14,15]$. For example, many secreted proteins in culture media can cause changes in secretion status even during the short-term serum starvation process [16]. The pretreatment approach can have a major impact on downstream analysis. Therefore, we did not perform serum starvation in our experiments. In order to reduce 


\section{ССТ8}
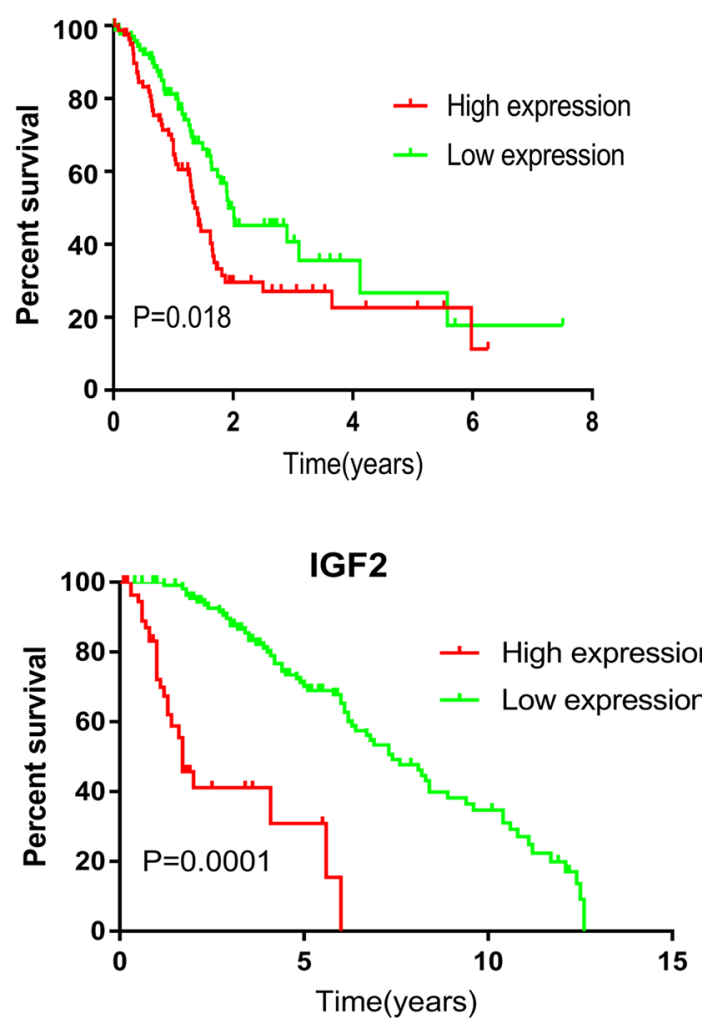

CTSL

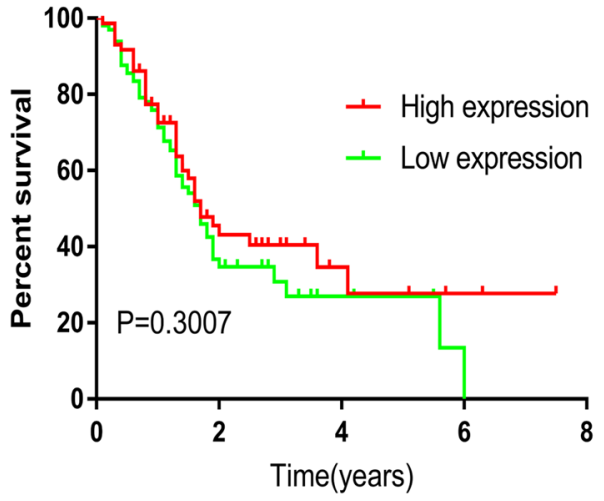

SAA1

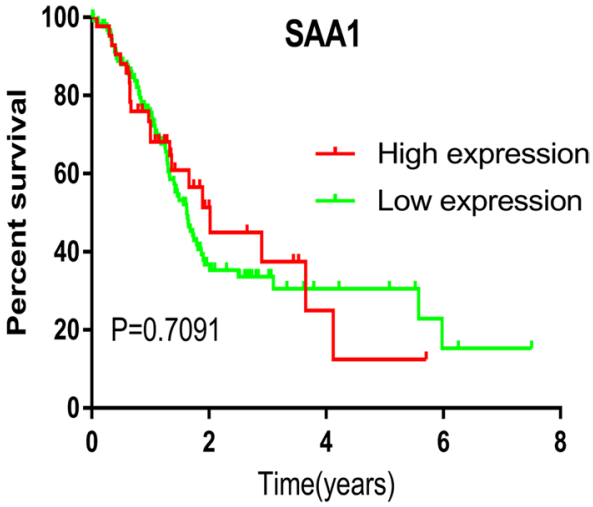

Fig. 4 Expression level and prognostic value of the differentially expressed secretome proteins in Human Protein Atlas database
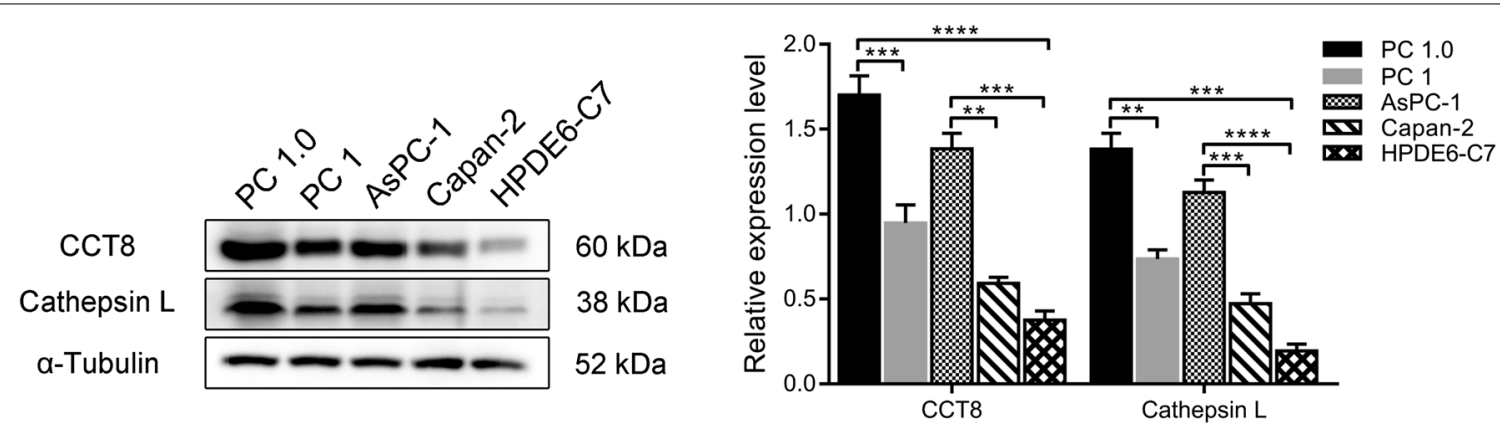

Fig. 5 Western blot validation of CCT8 and CTSL from PC-1.0, PC-1, AsPC-1, Capan-2 and HPDE6-C7 cell lines $(n=3)$

the effects of high-abundance proteins, the secreted proteins in the supernatant were separated using the SEC and H14 columns, and the results were thus more reliable (Fig. 8).Based on the results of previous experiments, cell biological changes can occur between pancreatic cancer cells with different capacities for invasion. The difference between the secretomes produced by two cell lines is very important for understanding the molecular mechanism of tumor cell invasion and metastasis.
Through GO and KEGG analysis, it was confirmed that the main functions of the differential proteins were concentrated in cell secretion, which increased the reliability of our experimental results. It is well known that tumor development and progression occurs over three stages, of which cellular cancerous transformation is the best stage for monitoring and treatment. Liquid biopsy can be used to observe the risk of carcinogenesis at the cellular level, provide early warning, and be used for screening 

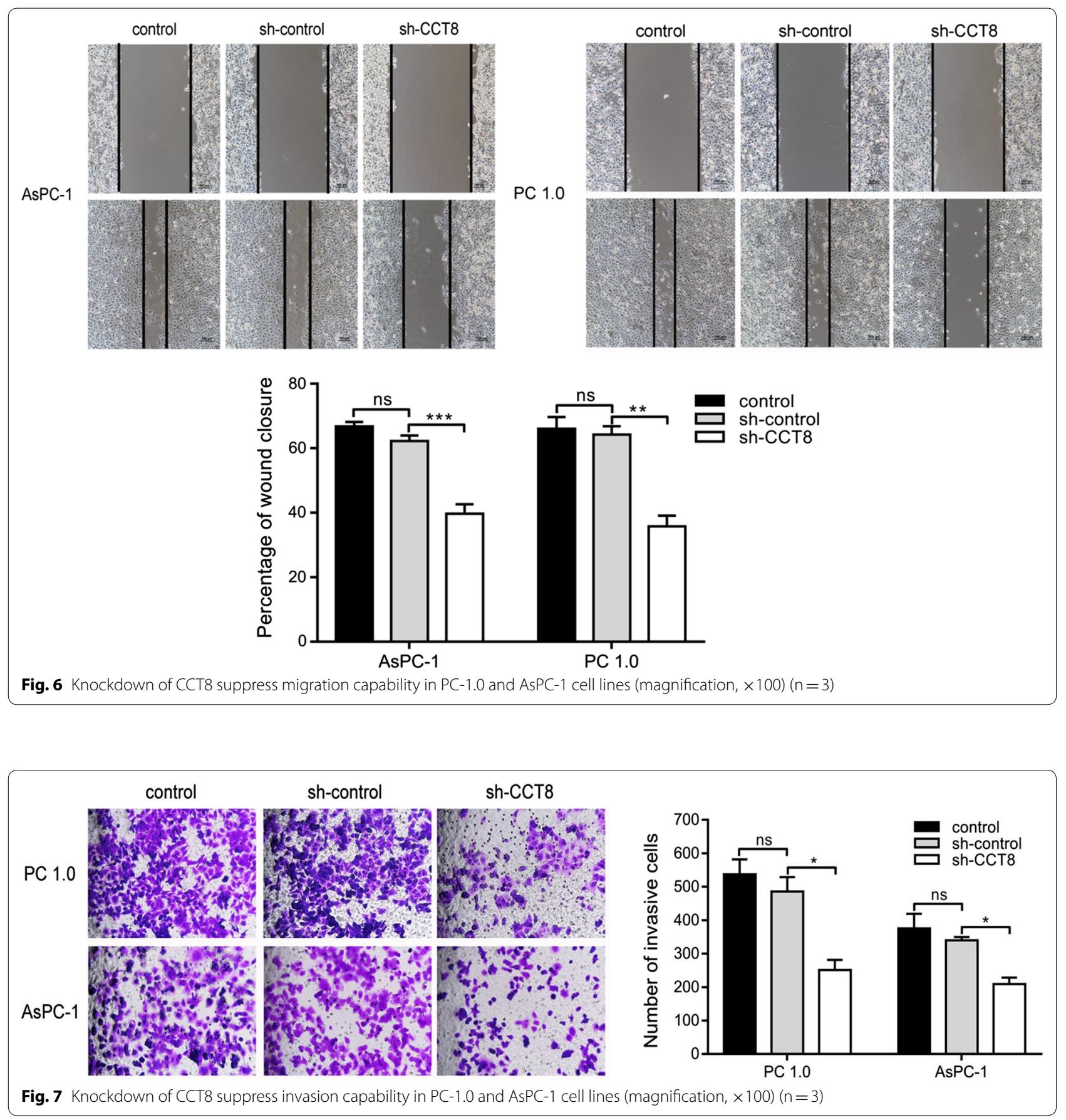

of high-risk populations. Traditional pathological biopsy has certain risks, and these tumor biomarkers derived from secreted proteins can be directly detected in body fluids, which can help distinguish between benign and malignant tumors earlier and more quickly, and help develop more appropriate treatment plans. In addition, they can also suggest the existence of occult micrometastases in the body. For patients with metastatic tumors without metastasis or undiscovered metastasis, it can help with more accurate staging and guide clinical selection of more appropriate treatment methods [17].

To summarize the results of this manuscript, we have identified a small number of proteins, but the literature and our own data confirm that these differential proteins are secreted via exosomes, which proves that the results of experiments are close to the actual status of secretion. 


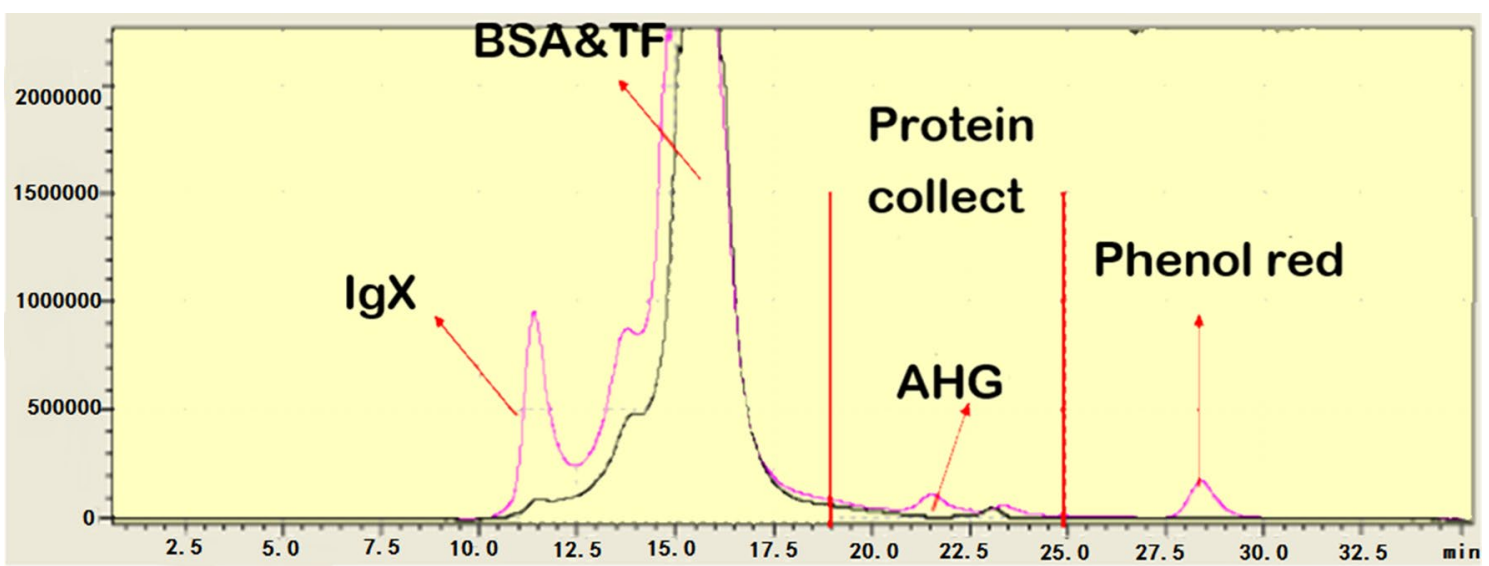

Fig. 8 The figure show that the collection time of sample fraction in chromatography

PPI network analysis showed that there was no direct relationship between these eight differential proteins. After data mining, we found that CCT8 is a potential core protein. Chaperonin-containing $\mathrm{T}$-complex protein $(\mathrm{CCT})$ has eight subunits, CCT1-8. The literature reports that $\mathrm{CCT} 8$ can affect invasion and metastasis of glioma cell carcinoma [18]. The TRic complex formed by CCT 8 and other proteins can mediate protein folding and play an essential role in the telomerase pathway [19]. Currently, the relationship between the function of CCT8 as a secreted protein and pancreatic cancer has not been reported in the literature. We found through survival analysis that CCT8 can be used as a predictor of pancreatic cancer. CTSL upregulation has been found in many cancers to be positively associated with cancer invasion and metastasis and poor prognosis [20-23]. In addition, it has been reported in the literature that CTSL is highly expressed in the plasma of patients with pancreatic cancer and can be used as an independent predictor of pancreatic cancer [24]. Toriola et al. detected the expression of circulating IGF2 in blood, which was confirmed to be associated with pancreatic cancer prognosis [25]. SAA1 is highly expressed in cancer-associated fibroblasts (CAFs), and the higher the expression of SAA1 in the matrix composition, the worse the prognosis. Selective inhibition of SAA1 expression in CAF may provide potential therapeutic benefit to patients with PDAC [26]. In addition, detection of SAA in the blood is useful for distinguishing between malignant and benign diseases as well as healthy controls [27]. Online database suggested that CTSL and SAA1 were not associated with pancreatic cancer prognosis $(p>0.05)$, probably due to changes in protein content during tissue sample processing. Further in vitro functional experiments showed that knockdown of CCT8 suppressed invasion and metastasis of pancreatic cancer cell lines. In future studies, we will perform functional analysis of CCT8in vivo, while collecting clinical blood samples for cohort studies.

\section{Conclusion}

In this study, samples were separated using two columns together in conjunction with dimethyl labeling, and mass spectrometry were used to improve the identification of secreted proteins under complete-medium conditions. Through biological analysis and cytological validation, it was confirmed that circulating CCT8 is a new predictive biomarker for pancreatic cancer.

\section{Supplementary information}

Supplementary information accompanies this paper at https://doi. org/10.1186/s12935-019-0980-1.

Additional file 1: Table S1. A complete list of all the proteins identified in this study.

Additional file 2: Table S2. The acquired raw files were analyzed by Maxquant. List of 12 proteins differentially expression by PC-1.0 and PC-1 pancreatic cancer cells with fold change $>1.5$.

Additional file 3: Table S3. GO term analysis of the identified proteins by DAVID.

Additional file 4: Table S4. String network coordinates.

Additional file 5: Table S5. The mRNA expression levels of 4 gene in pancreatic cancer were searched against the Human Protein Atlas data.

\section{Abbreviations}

CCT8: chaperonin-containing T-complex protein 8; CTSL: cathepsin L; IGF2: insulin like growth factor 2; GO: gene ontology; SAA1: serum amyloid A1; ACN: acetonitrile; BSA: bovine serum albumin; TFA: trifluoroacetic acid; SEC: size exclusion chromatography; BCA: bicinchoninic acid; FASP: filter-aidedsample preparation, CC: cellular component; BP: biological process; MF: molecular function; shRNA: short hairpin RNA; SDS-PAGE: sodium dodecyl sulfate polyacrylamide gel electrophoresis. 


\section{Acknowledgements \\ Not applicable.}

\section{Authors' contributions}

$\mathrm{PL}$ analyzed the data and wrote the paper. PL and HJ performed mass spectrometry analysis assay and analyzed the data. LK performed function assay. YW are responsible for culturing cells. XT and BS designed the experiment and revised the paper. All authors read and approved the final manuscript.

\section{Funding}

The present study were funded by Liaoning Provincial Natural Science Foundation Project (Grant Number: 180530068), Shenyang Technology Project (Grant Number: 17231178), and the Outstanding Young Doctor Fund of China Medical University (Grant Number: QGZD2018050).

\section{Availability of data and materials}

All data generated or analyzed during this study are included in this published article and its additional files.

\section{Ethics approval and consent to participate}

Not applicable.

\section{Consent for publication}

Not applicable.

\section{Competing interests}

The authors declare that they have no competing interests.

Received: 7 April 2019 Accepted: 27 September 2019 Published online: 11 October 2019

\section{References}

1. Siegel RL, Miller KD, Jemal A. Cancer statistics, 2019. CA Cancer J Clin. 2019;69(1):7-34

2. Zhou B, Xu JW, Cheng YG, Gao JY, Hu SY, Wang L, et al. Early detection of pancreatic cancer: Where are we now and where are we going? Int J Cancer. 2017;141(2):231-41.

3. Rofi E, Vivaldi C, Del Re M, Arrigoni E, Crucitta S, Funel N, et al. The emerging role of liquid biopsy in diagnosis, prognosis and treatment monitoring of pancreatic cancer. Pharmacogenomics. 2019;20(1):49-68.

4. Hanahan D, Weinberg RA. Hallmarks of cancer: the next generation. Cell. 2011;144(5):646-74

5. Lee YC, Gajdosik MS, Josic D, Clifton JG, Logothetis C, Yu-Lee LY, et al. Secretome analysis of an osteogenic prostate tumor identifies complex signaling networks mediating cross-talk of cancer and stromal cells within the tumor microenvironment. Mol Cell Proteomics. 2015;14(3):471-83.

6. Hirota M, Egami H, Corra S, Fujii H, Chaney WG, Rizzino A, et al. Production of scatter factor-like activity by a nitrosamine-induced pancreatic cancer cell line. Carcinogenesis. 1993;14(2):259-64.

7. Tan X, Liu P, Huang Y, Zhou L, Yang Y, Wang H, et al. Phosphoproteome analysis of invasion and metastasis-related factors in pancreatic cancer cells. PLoS ONE. 2016;11(3):e0152280.

8. da Huang W, Sherman BT, Lempicki RA. Systematic and integrative analysis of large gene lists using DAVID bioinformatics resources. Nat Protoc. 2009;4(1):44-57.

9. Szklarczyk D, Morris JH, Cook H, Kuhn M, Wyder S, Simonovic M, et al. The STRING database in 2017: quality-controlled protein-protein association networks, made broadly accessible. Nucleic Acids Res. 2017:45(D1):D362-8.
10. Keerthikumar S, Chisanga D, Ariyaratne D, Al Saffar H, Anand S, Zhao K, et al. ExoCarta: A Web-Based Compendium of Exosomal Cargo. J Mol Biol. 2016:428(4):688-92.

11. Uhlen M, Zhang C, Lee S, Sjostedt E, Fagerberg L, Bidkhori G, et al. A pathology atlas of the human cancer transcriptome. Science. 2017;357(6352):eaan2507.

12. Jin $H$, Liu $P$, Wu Y, Meng $X$, Wu M, Han J, et al. Exosomal zinc transporter ZIP4 promotes cancer growth and is a novel diagnostic biomarker for pancreatic cancer. Cancer Sci. 2018;109(9):2946-56.

13. Klemm F, Joyce JA. Microenvironmental regulation of therapeutic response in cancer. Trends Cell Biol. 2015;25(4):198-213.

14. Coumans FAW, Brisson AR, Buzas El, Dignat-George F, Drees EEE, ElAndaloussi S, et al. Methodological guidelines to study extracellular vesicles. Circ Res. 2017;120(10):1632-48

15. Brandi J, Manfredi M, Speziali G, Gosetti F, Marengo E, Cecconi D. Proteomic approaches to decipher cancer cell secretome. Semin Cell Dev Biol. 2018;78:93-101.

16. Eichelbaum K, Winter M, Berriel Diaz M, Herzig S, Krijgsveld J. Selective enrichment of newly synthesized proteins for quantitative secretome analysis. Nat Biotechnol. 2012:30(10):984-90.

17. Samandari M, Julia MG, Rice A, Chronopoulos A, Del Rio Hernandez AE. Liquid biopsies for management of pancreatic cancer. Transl Res. 2018;201:98-127

18. Qiu X, He X, Huang Q, Liu X, Sun G, Guo J, et al. Overexpression of CCT8 and its significance for tumor cell proliferation, migration and invasion in glioma. Pathol Res Pract. 2015;211(10):717-25

19. Freund A, Zhong FL, Venteicher AS, Meng Z, Veenstra TD, Frydman J, et al Proteostatic control of telomerase function through TRiC-mediated folding of TCAB1. Cell. 2014;159(6):1389-403.

20. Wang W, Long L, Wang L, Tan C, Fei X, Chen L, et al. Knockdown of cathepsin $\mathrm{L}$ promotes radiosensitivity of glioma stem cells both in vivo and in vitro. Cancer Lett. 2016;371(2):274-84.

21. Wang $L$, Zhao $Y$, Xiong $Y$, Wang W, Fei $Y$, Tan $C$, et al. K-ras mutation promotes ionizing radiation-induced invasion and migration of lung cancer in part via the cathepsin L/CUX1 pathway. Exp Cell Res. 2018:362(2):424-35.

22. Sudhan DR, Siemann DW. Cathepsin $L$ targeting in cancer treatment. Pharmacol Ther. 2015:155:105-16.

23. Lu HJ, Yan J, Jin PY, Zheng GH, Qin SM, Wu DM, et al. MicroRNA-152 inhibits tumor cell growth while inducing apoptosis via the transcriptional repression of cathepsin $L$ in gastrointestinal stromal tumor. Cancer Biomark. 2018:21(3):711-22.

24. Singh N, Das P, Gupta S, Sachdev V, Srivasatava S, Datta Gupta S, et al. Plasma cathepsin L: a prognostic marker for pancreatic cancer. World J Gastroenterol. 2014;20(46):17532-40.

25. Toriola AT, Ziegler M, Li Y, Pollak M, Stolzenberg-Solomon R. Prediagnosis circulating insulin-like growth factors and pancreatic cancer survival. Ann Surg Oncol. 2017;24(11):3212-9.

26. Djurec M, Grana O, Lee A, Troule K, Espinet E, Cabras L, et al. Saa3 is a key mediator of the protumorigenic properties of cancerassociated fibroblasts in pancreatic tumors. Proc Natl Acad Sci USA 2018;115(6):E1147-56.

27. Farooqui MS, Mittal A, Poudel B, Mall SK, Sathian B, Tarique M, et al. Improved diagnostic accuracy of pancreatic diseases with a combination of various novel serum biomarkers-case control study from Manipal Teaching Hospital, Pokhara, Nepal. Asian Pac J Cancer Prev. 2012;13(5):2171-4.

\section{Publisher's Note}

Springer Nature remains neutral with regard to jurisdictional claims in published maps and institutional affiliations. 